\title{
The Ulam Stability of Fractional Differential Equation with the Caputo-Fabrizio Derivative
}

\author{
Shuyi Wang \\ School of Mathematical Sciences, Qufu Normal University, Qufu, Shandong 273165, China \\ Correspondence should be addressed to Shuyi Wang; sywang_math@126.com
}

Received 11 November 2021; Accepted 14 December 2021; Published 7 January 2022

Academic Editor: Hüseyin Işık

Copyright (C) 2022 Shuyi Wang. This is an open access article distributed under the Creative Commons Attribution License, which permits unrestricted use, distribution, and reproduction in any medium, provided the original work is properly cited.

\begin{abstract}
The aim of this paper is to establish the Ulam stability of the Caputo-Fabrizio fractional differential equation with integral boundary condition. We also present the existence and uniqueness results of the solution for the Caputo-Fabrizio fractional differential equation by Krasnoselskii's fixed point theorem and Banach fixed point theorem. Some examples are provided to illustrate our theorems.
\end{abstract}

\section{Introduction}

Ulam [1] proposed to study the approximation degree of the approximate solution and the exact solution of the equation in 1940. Hyers [2] responded to Ulam's proposal and defined the Hyers-Ulam stability of equation in 1941. Later on, Rassias [3] extended Hyers's work and defined the Hyers-Ulam-Rassias stability of equation in 1978. The Hyers-Ulam stability and Hyers-Ulam-Rassias stability are collectively referred to as the Ulam stability. Subsequently, researchers initiated a research on the Ulam stability of integer-order differential equations (see [4-10]). Obloza [4], Cemil and Emel [5] proved the Hyers-Ulam stability and Hyers-Ulam-Rassias stability of the first-order differential equation, respectively. Wang et al. [6] studied the Ulam stability of the first-order differential equation with a boundary value condition. Otrocol and Ilea [7] obtained the Ulam stability of the first-order delay differential equation. Huang and $\mathrm{Li}$ [8] also obtained the Hyers-Ulam stability of another class of the first-order delay differential equation. Zada et al. [9] studied the Hyers-Ulam-Rassias stability of the higher order delay differential equation. However, the study on the Ulam stability of fractional differential equations is in its infancy.

Fractional differential equations are widely applied in physics [11, 12], control systems [13], chemical technology [14], and biosciences [15]. Fractional integral boundary value problems have been explored by many researchers. In particular, the integral boundary value problem provides a feasible method for the modeling of population dynamics and chemical engineering problems (see [16-18]). Although fractional integral boundary value problems are widely used, it is not easy to solve the equation, and the exact solution is often not obtained. Therefore, it is necessary to study the Ulam stability of fractional differential equations and use the approximate solution to replace the exact solution. So far, researchers have studied the Ulam stability and the existence and uniqueness of a solution for fractional differential equations with Hilfer-Hadamard, Caputo, and CaputoFabrizio fractional derivatives (see [19-22]). Abbas et al. [19] proved the existence and the Ulam stability of a fractional differential equation with the Hilfer-Hadamard derivative.

In [20], Wang et al. established the Ulam stability and data dependence for the Caputo fractional differential equation

$$
{ }^{c} D^{\beta} x(t)=k(t, x(t)), t \in[a,+\infty) .
$$

In [21], Dai et al. studied the Ulam stability of the Caputo fractional differential equation with an integral boundary condition 


$$
\left\{\begin{array}{l}
x^{\prime}(t)+{ }^{c} D_{0^{+}}^{\beta} x(t)=k(t, x(t)), t \in[0,1] \\
x(1)=I_{0^{+}}^{\gamma} x(\eta)
\end{array}\right.
$$

where $I_{0^{+}}^{\gamma}(\cdot)$ is the Riemann-Liouville fractional integral, $\gamma>0$.

In [22], Liu et al. obtained the Hyers-Ulam stability and the existence of solutions for the Caputo-Fabrizio fractional differential equation

$$
{ }^{C F} D^{\beta} x(t)=k(t, x(t)), t \in[0, T],
$$

where ${ }^{C F} D^{\beta}(\cdot)$ is the Caputo-Fabrizio fractional derivative, $\beta \in(0,1)$.

Motivated by [20-22], in this paper, our purpose is to study the existence and uniqueness of a solution and the Ulam stability of the following Caputo-Fabrizio fractional differential equation with boundary value condition:

$$
\left\{\begin{array}{l}
x^{\prime}(t)+{ }^{C F} D^{\beta} x(t)=k(t, x(t)), t \in[0,1] \\
x(1)=I_{0^{+}}^{\gamma} x(\xi)
\end{array}\right.
$$

where $x(t)$ is a continuous differentiable function on $[0,1]$; $k:[0,1] \times \mathbb{R} \longrightarrow \mathbb{R}$ is continuous; ${ }^{C F} D^{\beta}(\cdot)$ is the CaputoFabrizio fractional derivative, $\beta \in(0,1)$; and $I_{0^{+}}^{\gamma}(\cdot)$ is the Riemann-Liouville fractional integral, $\gamma>0, \xi \in[0,1]$.

Equation (4) is a new kind of the Korteweg-de VriesBergers (KDVB) equation model. In [23], Equation (4) is used to describe unusual irregularities and nonlinearities in wave dynamics and liquids motions.

The main contributions are as follows: Firstly, we give the definitions of the Hyers-Ulam stability and HyersUlam-Rassias stability for Equation (4). Then, we obtain a sufficient condition to derive the uniqueness of the solution for Equation (4) by the Banach contraction principle. Next, we give a sufficient condition to prove the existence of the solution for Equation (4) by Krasnoselskii's fixed point theorem. On this basis, we give the Ulam stability results for Equation (4) by the Laplace transform and inequality results.

The rest of our article is arranged as follows. Some basic definitions and necessary theorems are presented in Section 2. We establish sufficient conditions to show existence and uniqueness of solution for the Caputo-Fabrizio fractional differential equation in Section 3. In Section 4, we prove the Ulam stability of the Caputo-Fabrizio fractional differential equation. Two examples are provided in Section 5 to illustrate our theorems.

\section{Preliminaries}

We will denote by $C^{1}[0,1]$ the space of continuous differentiable functions on $[0,1]$ with norm

$$
\|x\|=\sup \{|x(t)|, t \in[0,1]\} .
$$

Definition 1 [24]. The Caputo-Fabrizio fractional derivative of order $\beta$ of a continuous differentiable function $x$ is given by

$$
{ }^{C F} D^{\beta} x(t)=\frac{(2-\beta) M(\beta)}{2(1-\beta)} \int_{0}^{t} \exp \left(-\frac{\beta}{1-\beta}(t-\tau)\right) x^{\prime}(\tau) d \tau, t \geq 0,
$$

the normalization function $M(\beta)$ depends on $\beta$.

Definition 2 [25]. The Riemann-Liouville fractional integral of order $\gamma$ of a function $x$ is given by

$$
I_{0^{+}}^{\gamma} x(t)=\frac{1}{\Gamma(\gamma)} \int_{0}^{t}(t-\tau)^{\gamma-1} x(\tau) d \tau, t \geq 0 .
$$

Based on Definition 2 in [5] and Definition 2.1 in [9], we give the definitions of the Hyers-Ulam stability and the Hyers-Ulam-Rassias stability for Equation (4).

Definition 3. Equation (4) has the Hyers-Ulam stability if and only if for any solution $x(t)$ of

$$
\left|x^{\prime}(t)+{ }^{C F} D^{\beta} x(t)-k(t, x(t))\right| \leq \varepsilon, t \in[0,1],
$$

where $\varepsilon>0$, there is a constant $C>0$ and a solution $y(t)$ of Equation (4) satisfying

$$
|x(t)-y(t)| \leq C \times \varepsilon, t \in[0,1] .
$$

Definition 4. Equation (4) has the Hyers-Ulam-Rassias stability if and only if for any solution $x(t)$ of

$$
\left|x^{\prime}(t)+{ }^{C F} D^{\beta} x(t)-k(t, x(t))\right| \leq \delta(t), t \in[0,1],
$$

where $\delta(t) \in C\left([0,1], R_{+}\right)$, there is a constant $K_{k, \delta}>0$ and a solution $y(t)$ of Equation (4) satisfying

$$
|x(t)-y(t)| \leq K_{k, \delta} \times \delta(t), t \in[0,1]
$$

Theorem 5 [26]. If $x$ is a piecewise continuous function and there exist $K>0$ and $\mu$ such that

$$
|x(t)| \leq K e^{\mu t}, t \geq t_{0}
$$

then the Laplace transform $L[x(t)](s)$ exists.

Theorem 6 [27]. Let $\beta \in(0,1)$. The Laplace transform of ${ }^{C F} D^{\beta} x(t)$ is

$$
L\left[{ }^{C F} D^{\beta} x(t)\right](s)=\frac{(2-\beta) M(\beta)}{2(s+\beta(1-s))}(s L[x(t)](s)-x(0)), s>0,
$$

where $L[x(t)](s)$ is the Laplace transform of $x(t)$. 
Theorem 7. The solution of the following fractional problem

$$
\left\{\begin{array}{l}
x^{\prime}(t)+{ }^{C F} D^{\beta} x(t)=k(t, x(t)), t \in[0,1] \\
x(1)=I_{0^{+}}^{\gamma} x(\xi)
\end{array}\right.
$$

is given by

$$
x(t)=I_{0^{+}}^{\gamma} x(\xi)+\int_{0}^{1} G(t, s) k(s, x(s)) d s,
$$

where

$$
G(t, s)= \begin{cases}\left(1-\frac{b_{\beta}}{a_{\beta}}\right) \exp \left(-a_{\beta}(t-s)\right)-\left(1-\frac{b_{\beta}}{a_{\beta}}\right) \exp \left(-a_{\beta}(1-s)\right), & 0 \leq s \leq t, \\ -\left(1-\frac{b_{\beta}}{a_{\beta}}\right) \exp \left(-a_{\beta}(1-s)\right)-\frac{b_{\beta}}{a_{\beta}}, & t \leq s \leq 1,\end{cases}
$$

$$
a_{\beta}=\frac{2 \beta+(2-\beta) M(\beta)}{2(1-\beta)}, b_{\beta}=\frac{2 \beta}{2(1-\beta)} .
$$

Proof. Since $x(t)$ is continuous differentiable function on $[0,1], x^{\prime}(t)$ is bounded function on $[0,1]$. By Definition 1 , ${ }^{C F} D^{\beta} x(t)$ is also a bounded function. Then, there exist constants $k_{1}, k_{2}>0$ and $\mu_{1}, \mu_{2}$ such that

$$
\begin{gathered}
\left|x^{\prime}(t)\right| \leq k_{1} e^{\mu_{1} t}, t \geq t_{0}>0, \\
\left|{ }^{C F} D^{\beta} x(t)\right| \leq k_{2} e^{\mu_{2} t}, t \geq t_{0}>0 .
\end{gathered}
$$

From Theorem 5, the Laplace transform of $x^{\prime}(t)$ and ${ }^{C F} D^{\beta} x(t)$ exists.

Taking the Laplace transform for the first formula of Equation (14), we conclude

$$
s \tilde{x}(s)-x(0)+\frac{(2-\beta) M(\beta)}{2(s+\beta(1-s))}(s \tilde{x}(s)-x(0))=\tilde{k}(s, x(s)),
$$

or

$$
\begin{aligned}
\tilde{x}(s)= & \frac{1}{s} x(0)+\frac{1}{s+(2 \beta+(2-\beta) M(\beta) / 2(1-\beta))} \tilde{k}(s, x(s)) \\
& +\frac{2 \beta / 2(1-\beta)}{s(s+(2 \beta+(2-\beta) M(\beta) / 2(1-\beta)))} \tilde{k}(s, x(s)) .
\end{aligned}
$$

Taking the Laplace inverse transform for the above equation, we conclude

$$
\begin{aligned}
x(t)= & x(0)+\left(1-\frac{b_{\beta}}{a_{\beta}}\right) \int_{0}^{t} \exp \left(-a_{\beta}(t-s)\right) k(s, x(s)) d s \\
& +\frac{b_{\beta}}{a_{\beta}} \int_{0}^{t} k(s, x(s)) d s .
\end{aligned}
$$

Then

$$
\begin{aligned}
x(1)= & x(0)+\left(1-\frac{b_{\beta}}{a_{\beta}}\right) \int_{0}^{1} \exp \left(-a_{\beta}(1-s)\right) k(s, x(s)) d s \\
& +\frac{b_{\beta}}{a_{\beta}} \int_{0}^{1} k(s, x(s)) d s .
\end{aligned}
$$

Since $x(1)=I_{0^{+}}^{\gamma} x(\xi)$, thus

$$
\begin{aligned}
x(0)= & I_{0^{+}}^{\gamma} x(\xi)-\left(1-\frac{b_{\beta}}{a_{\beta}}\right) \int_{0}^{1} \exp \left(-a_{\beta}(1-s)\right) k(s, x(s)) d s \\
& -\frac{b_{\beta}}{a_{\beta}} \int_{0}^{1} k(s, x(s)) d s .
\end{aligned}
$$

Then

$$
\begin{aligned}
x(t)= & I_{0^{+}}^{\gamma} x(\xi)-\left(1-\frac{b_{\beta}}{a_{\beta}}\right) \int_{0}^{1} \exp \left(-a_{\beta}(1-s)\right) k(s, x(s)) d s \\
& -\frac{b_{\beta}}{a_{\beta}} \int_{0}^{1} k(s, x(s)) d s+\left(1-\frac{b_{\beta}}{a_{\beta}}\right) \int_{0}^{t} \exp \\
& \cdot\left(-a_{\beta}(t-s)\right) k(s, x(s)) d s+\frac{b_{\beta}}{a_{\beta}} \int_{0}^{t} k(s, x(s)) d s .
\end{aligned}
$$

By the definition of $G(t, s)$, we conclude

$$
x(t)=I_{0^{+}}^{\gamma} x(\xi)+\int_{0}^{1} G(t, s) k(s, x(s)) d s .
$$

Remark 8 .

$$
\begin{aligned}
\int_{0}^{t}|G(t, s)| d s= & \int_{0}^{t} \mid\left(1-\frac{b_{\beta}}{a_{\beta}}\right) \exp \left(-a_{\beta}(t-s)\right) \\
& -\left(1-\frac{b_{\beta}}{a_{\beta}}\right) \exp \left(-a_{\beta}(1-s)\right) \mid d s \\
\leq & \int_{0}^{t}\left[\left(1-\frac{b_{\beta}}{a_{\beta}}\right) \exp \left(-a_{\beta}(t-s)\right)\right. \\
& \left.+\left(1-\frac{b_{\beta}}{a_{\beta}}\right) \exp \left(-a_{\beta}(1-s)\right)\right] d s \\
\leq & \int_{0}^{t}\left[\exp \left(-a_{\beta}(t-s)\right)+\exp \left(-a_{\beta}(1-s)\right)\right] d s \\
= & {\left[\exp \left(-a_{\beta} t\right)+\exp \left(-a_{\beta}\right)\right] \cdot \int_{0}^{t} \exp \left(a_{\beta} s\right) d s } \\
= & \frac{1}{a_{\beta}} \cdot\left[\exp \left(-a_{\beta} t\right)+\exp \left(-a_{\beta}\right)\right] \\
& \cdot\left[\exp \left(a_{\beta} t\right)-1\right] \leq \frac{2}{a_{\beta}} \cdot\left[1-\exp \left(-a_{\beta}\right)\right]=E .
\end{aligned}
$$


Thus, there exists a constant $E>0$ such that

$$
\int_{0}^{t}|G(t, s)| d s \leq E, t \in[0,1]
$$

Theorem 9 (Krasnoselskii's fixed point theorem). Let $S$ be a bounded convex closed subset of a Banach space $W$, and $P$, $Q: S \longrightarrow W$ satisfy the following:

(i) $P x+Q y \in S$, for all $x, y \in S$

(ii) $P$ is completely continuous

(iii) $Q$ is a contraction mapping

Then, $P+Q$ has at least one fixed point.

\section{Existence and Uniqueness Theorems for Fractional Differential Equation}

The following assumption will be needed throughout the paper:

$\left(S_{1}\right): k:[0,1] \times \mathbb{R} \longrightarrow \mathbb{R}$ is a continuous function.

$\left(S_{2}\right): k(t, x)$ satisfies the following Lipschitz condition for the second variable:

$$
\left|k\left(t, x_{1}\right)-k\left(t, x_{2}\right)\right| \leq c_{k}\left|x_{1}-x_{2}\right|, x_{1}, x_{2} \in \mathbb{R}, t \in[0,1] .
$$

$\left(S_{3}\right)$ : Let $\delta(t):[0,1] \longrightarrow \mathbb{R}_{+}$satisfy

$$
\int_{0}^{t} \delta(s) d s \leq L_{\delta} \cdot \delta(t), L_{\delta}>0, t \in[0,1] .
$$

Theorem 10. Suppose that $\left(S_{1}\right)$ and $\left(S_{2}\right)$ are satisfied; then Equation (4) has a unique solution provided that $\xi^{\gamma}$ / $(\Gamma(\gamma+1))+E c_{k}<1$.

Proof. Since $k \in C([0,1] \times \mathbb{R}, \mathbb{R})$, there exists $T>0$ such that

$$
T=\max _{t \in[0,1], s \in \mathbb{R}}|k(t, s)|
$$

Similar to the proof of Theorem 3 in [22]. Let operator $F$ be given by

$$
(F x)(t)=I_{0^{+}}^{\gamma} x(\xi)+\int_{0}^{1} G(t, s) k(s, x(s)) d s .
$$

Firstly, we prove that $F$ maps a closed set into a closed set.
Let $U_{b}=\left\{x \in C^{1}([0,1], \mathbb{R}) \mid\|x\| \leq b, b \geq E T / 1-\xi^{\gamma} / \Gamma(\gamma\right.$ $+1)>0\}$. For $x \in U_{b}$, it follows that

$$
\begin{aligned}
|(F x)(t)| & \leq \frac{1}{\Gamma(\gamma)} \int_{0}^{\xi}(\xi-s)^{\gamma-1}|x(s)| d s+\int_{0}^{1}|G(t, s)||k(s, x(s))| d s \\
& \leq \frac{\xi^{\gamma}}{\Gamma(\gamma+1)} b+E T \leq b .
\end{aligned}
$$

This implies $F U_{b} \subseteq U_{b}$.

Then, we prove that $F$ is a strict contraction.

Let $x_{1}, x_{2} \in C^{1}([0,1], \mathbb{R})$, for any $t \in[0,1]$; it follows that

$$
\begin{aligned}
\left|\left(F x_{1}\right)(t)-\left(F x_{2}\right)(t)\right| \leq & \mid \frac{1}{\Gamma(\gamma)} \int_{0}^{\xi}(\xi-s)^{\gamma-1}\left(x_{1}(s)-x_{2}(s)\right) d s \\
& +\int_{0}^{1} G(t, s)\left(k\left(s, x_{1}(s)\right)-k\left(s, x_{2}(s)\right)\right) d s \mid \\
\leq & \left(\frac{\xi^{\gamma}}{\Gamma(\gamma+1)}+E c_{k}\right)\left\|x_{1}-x_{2}\right\| .
\end{aligned}
$$

As $\xi^{\gamma} /(\Gamma(\gamma+1))+E c_{k}<1$, for $x_{1}, x_{2} \in C^{1}([0,1], \mathbb{R}), F$ is a strict contraction. From the Banach fixed point theorem, $F$ has a unique fixed point $x^{*}(t) \in C^{1}([0,1], \mathbb{R})$; accordingly, Equation (4) has a unique solution.

Theorem 11. Suppose that $\left(S_{1}\right)$ and $\left(S_{2}\right)$ are satisfied; then Equation (4) has at least one solution provided that $\xi^{\gamma} /(\Gamma$ $(\gamma+1))+E c_{k}<1$.

Proof. Since $k \in C([0,1] \times \mathbb{R}, \mathbb{R})$, there exists $T>0$ such that

$$
T=\max _{t \in[0,1], s \in \mathbb{R}}|k(t, s)| .
$$
$0\}$.

Let $U_{c}=\left\{x \in C^{1}[0,1] \mid\|x\| \leq c, c \geq E T / 1-\xi^{\gamma} / \Gamma(\gamma+1)>\right.$ Let operators $P$ and $Q$ be given by

$$
\begin{aligned}
(P x)(t)= & -\left(1-\frac{b_{\beta}}{a_{\beta}}\right) \int_{t}^{1} \exp \left(-a_{\beta}(1-s)\right) k(s, x(s)) d s \\
& -\frac{b_{\beta}}{a_{\beta}} \int_{t}^{1} k(s, x(s)) d s,
\end{aligned}
$$

$$
\begin{aligned}
(Q x)(t)= & I_{0^{+}}^{\gamma} x(\xi)+\left(1-\frac{b_{\beta}}{a_{\beta}}\right) \int_{0}^{t} \exp \left(-a_{\beta}(t-s)\right) k(s, x(s)) \\
& -\exp \left(-a_{\beta}(1-s)\right) k(s, x(s)) d s .
\end{aligned}
$$


Firstly, for all $x_{1}, x_{2} \in U_{c}$, using Remark 8 , it follows that

$$
\begin{aligned}
\left\|P x_{1}+Q x_{2}\right\|= & \sup \mid-\left(1-\frac{b_{\beta}}{a_{\beta}}\right) \int_{t}^{1} \exp \left(-a_{\beta}(1-s)\right) \\
& \cdot k\left(s, x_{1}(s)\right) d s-\frac{b_{\beta}}{a_{\beta}} \int_{t}^{1} k\left(s, x_{1}(s)\right) d s+I_{0^{+}}^{\gamma} x_{2}(\xi) \\
& +\left(1-\frac{b_{\beta}}{a_{\beta}}\right) \int_{0}^{t} \exp \left(-a_{\beta}(t-s)\right) k\left(s, x_{2}(s)\right) \\
& -\exp \left(-a_{\beta}(1-s)\right) k\left(s, x_{2}(s)\right) d s \mid \\
\leq & \sup \mid \int_{0}^{t} G(t, s) k\left(s, x_{2}(s)\right) d s \\
& +\int_{t}^{1} G(t, s) k\left(s, x_{1}(s)\right) d s \mid \\
& +\sup \left|\frac{1}{\Gamma(\gamma)} \int_{0}^{\xi}(\xi-s)^{\gamma-1} x_{2}(s) d s\right| \\
\leq & \sup \left\{\int_{0}^{t}|G(t, s)|\left|k\left(s, x_{2}(s)\right)\right| d s\right. \\
& \left.+\int_{t}^{1}|G(t, s)|\left|k\left(s, x_{1}(s)\right)\right| d s\right\} \\
& +\sup \left|\frac{1}{\Gamma(\gamma)} \int_{0}^{\xi}(\xi-s)^{\gamma-1} d s\right| \cdot c \\
\leq & \sup \left\{\int_{0}^{1}|G(t, s)| d s\right\} \cdot T+\frac{\xi^{\gamma}}{\Gamma(\gamma+1)} c \\
& \xi^{\gamma}(\gamma+1) c+E T \leq c . \\
&
\end{aligned}
$$

Hence, we have $P x_{1}+Q x_{2} \in U_{c}$.

Then, for all $x_{1}, x_{2} \in C^{1}[0,1]$,

$$
\begin{aligned}
\left\|Q x_{1}-Q x_{2}\right\|= & \sup \mid I_{0^{+}}^{\gamma} x_{1}(\xi)-I_{0^{+}}^{\gamma} x_{2}(\xi)+\left(1-\frac{b_{\beta}}{a_{\beta}}\right) \int_{0}^{t} \\
& \cdot\left[\exp \left(-a_{\beta}(t-s)\right)-\exp \left(-a_{\beta}(1-s)\right)\right] \\
& \cdot\left[k\left(s, x_{1}(s)\right)-k\left(s, x_{2}(s)\right)\right] d s \mid \\
\leq & \sup \left|\frac{1}{\Gamma(\gamma)} \int_{0}^{\xi}(\xi-s)^{\gamma-1}\right| x_{1}(s)-x_{2}(s)|d s| \\
& +\sup \left|\int_{0}^{t}\right| G(t, s)|\cdot| k\left(s, x_{1}(s)\right)-k\left(s, x_{2}(s)\right)|d s| \\
\leq & \left(\frac{\xi^{\gamma}}{\Gamma(\gamma+1)}+E c_{k}\right)\left\|x_{1}-x_{2}\right\| .
\end{aligned}
$$

As $\xi^{\gamma} /(\Gamma(\gamma+1))+E c_{k}<1, Q$ is a contraction mapping. Finally, we prove operator $P$ is completely continuous.
Step 1. Operator $P$ is continuous.

Let $x_{n}$ be a convergent sequence, $x_{n} \longrightarrow x \in C^{1}([0,1], \mathbb{R})$, by Remark 8 and $\left(S_{2}\right)$; it follows that

$$
\begin{aligned}
\left|\left(P x_{n}\right)(t)-(P x)(t)\right|=\mid & \mid\left(1-\frac{b_{\beta}}{a_{\beta}}\right) \int_{t}^{1} \exp \left(-a_{\beta}(1-s)\right)\left(k\left(s, x_{n}(s)\right)\right. \\
& -k(s, x(s))) d s+\frac{b_{\beta}}{a_{\beta}} \int_{t}^{1}\left(k\left(s, x_{n}(s)\right)\right. \\
& -k(s, x(s))) d s\left|\leq \int_{t}^{1}\right| G(t, s) \| k\left(s, x_{n}(s)\right) \\
& -k(s, x(s)) \mid d s \leq E c_{k}\left\|x_{n}-x\right\| .
\end{aligned}
$$

Since $x_{n} \longrightarrow x$, we have $P x_{n} \longrightarrow P x$; then operator $P$ is continuous.

Step 2. Operator $P$ is bounded on $U_{c}$.

$$
\begin{aligned}
|(P x)(t)|= & \mid-\left(1-\frac{b_{\beta}}{a_{\beta}}\right) \int_{t}^{1} \exp \left(-a_{\beta}(1-s)\right) k(s, x(s)) d s \\
& -\frac{b_{\beta}}{a_{\beta}} \int_{t}^{1} k(s, x(s)) d s|=| \int_{t}^{1} G(t, s) k(s, x(s)) d s \mid \\
\leq & \int_{t}^{1}|G(t, s)||k(s, x(s))| d s \leq E T .
\end{aligned}
$$

Step 3. Operator $P$ is equicontinuous in $C^{1}([0,1], \mathbb{R})$. Let $t_{1}, t_{2} \in[0,1]$ and $t_{2}<t_{1}, x \in U_{c}$; it follows that

$$
\begin{aligned}
\left|(P x)\left(t_{1}\right)-(P x)\left(t_{2}\right)\right|=\mid & \left(1-\frac{b_{\beta}}{a_{\beta}}\right) \int_{t_{2}}^{t_{1}} \exp \left(-a_{\beta}(1-s)\right) k(s, x(s)) d s \\
& +\frac{b_{\beta}}{a_{\beta}} \int_{t_{2}}^{t_{1}} k(s, x(s)) d s \mid \\
\leq & {\left[\left(1-\frac{b_{\beta}}{a_{\beta}}\right) \int_{t_{2}}^{t_{1}}\left|\exp \left(-a_{\beta}(1-s)\right)\right| d s+\frac{b_{\beta}}{a_{\beta}} \int_{t_{2}}^{t_{1}} d s\right] } \\
& \cdot T \leq T \cdot\left|t_{1}-t_{2}\right| .
\end{aligned}
$$

Then, operator $P$ is equicontinuous.

From Step 1-Step 3 and the Arzela-Ascoli theorem, $P$ is completely continuous. By Theorem 9, $P+Q$ has at least one fixed point, since

$$
(P x+Q x)(t)=I_{0^{+}}^{\gamma} x(\xi)+\int_{0}^{1} G(t, s) k(s, x(s)) d s .
$$

From Theorem 7, Equation (4) has at least one solution.

\section{Stability Results}

Theorem 12. Suppose that $\left(S_{1}\right)$ and $\left(S_{2}\right)$ are satisfied; then Equation (4) has the Hyers-Ulam stability on $[0,1]$. 
Proof. Since $\left(S_{1}\right)$ and $\left(S_{2}\right)$ hold, by Theorems 10 and 11, Equation (4) has a unique solution. From Theorem 7, Equation (4) has the unique solution

$$
\begin{aligned}
x(t)= & x(0)+\left(1-\frac{b_{\beta}}{a_{\beta}}\right) \int_{0}^{t} \exp \left(-a_{\beta}(t-s)\right) k(s, x(s)) d s \\
& +\frac{b_{\beta}}{a_{\beta}} \int_{0}^{t} k(s, x(s)) d s .
\end{aligned}
$$

Let $y(t)$ satisfy $y(0)=x(0)$ and be a solution of the inequality

$$
\left|y^{\prime}(t)+{ }^{C F} D^{\beta} y(t)-k(t, y(t))\right| \leq \varepsilon, t \in[0,1] .
$$

Set

$$
G(t)=y^{\prime}(t)+{ }^{C F} D^{\beta} y(t)-k(t, y(t)), t \in[0,1] .
$$

Then

$$
\begin{gathered}
y^{\prime}(t)+{ }^{C F} D^{\beta} y(t)=G(t)+k(t, y(t)), t \in[0,1], \\
|G(t)| \leq \varepsilon, t \in[0,1] .
\end{gathered}
$$

From the proof of Theorem 7, we conclude

$$
\begin{aligned}
y(t)= & y(0)+\left(1-\frac{b_{\beta}}{a_{\beta}}\right) \int_{0}^{t} \exp \left(-a_{\beta}(t-s)\right)[G(s)+k(s, y(s))] d s \\
& +\frac{b_{\beta}}{a_{\beta}} \int_{0}^{t}[G(s)+k(s, y(s))] d s .
\end{aligned}
$$

Then

$$
\begin{aligned}
& \mid y(t)-y(0)-\left(1-\frac{b_{\beta}}{a_{\beta}}\right) \int_{0}^{t} \exp \left(-a_{\beta}(t-s)\right) k(s, y(s)) d s \\
& \quad-\frac{b_{\beta}}{a_{\beta}} \int_{0}^{t} k(s, y(s)) d s|=|\left(1-\frac{b_{\beta}}{a_{\beta}}\right) \int_{0}^{t} \exp \left(-a_{\beta}(t-s)\right) G(s) d s \\
& \quad+\frac{b_{\beta}}{a_{\beta}} \int_{0}^{t} G(s) d s\left|\leq\left(1-\frac{b_{\beta}}{a_{\beta}}\right) \int_{0}^{t}\right| G(s)\left|d s+\frac{b_{\beta}}{a_{\beta}} \int_{0}^{t}\right| G(s) \mid d s \\
& \leq \int_{0}^{t}|G(s)| d s \leq \varepsilon .
\end{aligned}
$$

Thus

$$
\begin{aligned}
|y(t)-x(t)|= & \mid y(t)-x(0)-\left(1-\frac{b_{\beta}}{a_{\beta}}\right) \int_{0}^{t} \exp \\
& \cdot\left(-a_{\beta}(t-s)\right) k(s, x(s)) d s-\frac{b_{\beta}}{a_{\beta}} \int_{0}^{t} k(s, x(s)) d s \mid \\
\leq & \mid y(t)-y(0)-\left(1-\frac{b_{\beta}}{a_{\beta}}\right) \int_{0}^{t} \exp \\
& \cdot\left(-a_{\beta}(t-s)\right) k(s, y(s)) d s-\frac{b_{\beta}}{a_{\beta}} \int_{0}^{t} k(s, y(s)) d s \mid \\
& +\left(1-\frac{b_{\beta}}{a_{\beta}}\right) \int_{0}^{t} \exp \left(-a_{\beta}(t-s)\right) \mid k(s, y(s)) \\
& -k(s, x(s))\left|d s+\frac{b_{\beta}}{a_{\beta}} \int_{0}^{t}\right| k(s, y(s))-k(s, x(s)) \mid d s \\
\leq & \varepsilon+c_{k} \int_{0}^{t}|y(s)-x(s)| d s .
\end{aligned}
$$

From the Gronwall-Bellman inequality, we conclude

$$
|y(t)-x(t)| \leq\left[\exp \left(\int_{0}^{t} c_{k} d s\right)\right] \cdot \varepsilon \leq \exp \left(c_{k}\right) \cdot \varepsilon
$$

From Definition 3, Equation (4) has the Hyers-Ulam stability.

Theorem 13. Suppose that $\left(S_{1}\right),\left(S_{2}\right)$, and $\left(S_{3}\right)$ are satisfied; then Equation (4) has the Hyers-Ulam-Rassias stability on $[0,1]$.

Proof. Since $\left(S_{1}\right)$ and $\left(S_{2}\right)$ hold, by Theorems 10 and 11, Equation (4) has a unique solution. From Theorem 7, Equation (4) has the unique solution

$$
\begin{aligned}
x(t)= & x(0)+\left(1-\frac{b_{\beta}}{a_{\beta}}\right) \int_{0}^{t} \exp \left(-a_{\beta}(t-s)\right) k(s, x(s)) d s \\
& +\frac{b_{\beta}}{a_{\beta}} \int_{0}^{t} k(s, x(s)) d s .
\end{aligned}
$$

Let $y(t)$ satisfy $y(0)=x(0)$ and be a solution of the inequality

$$
\left|y^{\prime}(t)+{ }^{C F} D^{\beta} y(t)-k(t, y(t))\right| \leq \delta(t), t \in[0,1] .
$$

Set

$$
G(t)=y^{\prime}(t)+{ }^{C F} D^{\beta} y(t)-k(t, y(t)), t \in[0,1] .
$$


Then

$$
\begin{gathered}
y^{\prime}(t)+{ }^{C F} D^{\beta} y(t)=G(t)+k(t, y(t)), t \in[0,1] \\
|G(t)| \leq \delta(t), t \in[0,1] .
\end{gathered}
$$

From the proof of Theorem 7, we conclude

$$
\begin{aligned}
y(t)= & y(0)+\left(1-\frac{b_{\beta}}{a_{\beta}}\right) \int_{0}^{t} \exp \left(-a_{\beta}(t-s)\right)[G(s)+k(s, y(s))] d s \\
& +\frac{b_{\beta}}{a_{\beta}} \int_{0}^{t}[G(s)+k(s, y(s))] d s .
\end{aligned}
$$

Then by $\left(S_{3}\right)$, it follows that

$$
\begin{aligned}
& \mid y(t)-y(0)-\left(1-\frac{b_{\beta}}{a_{\beta}}\right) \int_{0}^{t} \exp \left(-a_{\beta}(t-s)\right) k(s, y(s)) d s \\
& -\frac{b_{\beta}}{a_{\beta}} \int_{0}^{t} k(s, y(s)) d s|=|\left(1-\frac{b_{\beta}}{a_{\beta}}\right) \int_{0}^{t} \exp \left(-a_{\beta}(t-s)\right) G(s) d s \\
& \quad+\frac{b_{\beta}}{a_{\beta}} \int_{0}^{t} G(s) d s\left|\leq\left(1-\frac{b_{\beta}}{a_{\beta}}\right) \int_{0}^{t}\right| G(s)\left|d s+\frac{b_{\beta}}{a_{\beta}} \int_{0}^{t}\right| G(s) \mid d s \\
& \leq \int_{0}^{t}|G(s)| d s \leq \int_{0}^{t} \delta(s) d s \leq L_{\delta} \cdot \delta(t) .
\end{aligned}
$$

Thus

$$
\begin{aligned}
& |y(t)-x(t)|=\mid y(t)-x(0)-\left(1-\frac{b_{\beta}}{a_{\beta}}\right) \int_{0}^{t} \exp \left(-a_{\beta}(t-s)\right) k(s, x(s)) d s \\
& -\frac{b_{\beta}}{a_{\beta}} \int_{0}^{t} k(s, x(s)) d s|\leq| y(t)-y(0)-\left(1-\frac{b_{\beta}}{a_{\beta}}\right) \int_{0}^{t} \exp \\
& \cdot\left(-a_{\beta}(t-s)\right) k(s, y(s)) d s-\frac{b_{\beta}}{a_{\beta}} \int_{0}^{t} k(s, y(s)) d s \mid \\
& +\left(1-\frac{b_{\beta}}{a_{\beta}}\right) \int_{0}^{t} \exp \left(-a_{\beta}(t-s)\right)|k(s, y(s))-k(s, x(s))| d s \\
& +\frac{b_{\beta}}{a_{\beta}} \int_{0}^{t}|k(s, y(s))-k(s, x(s))| d s \leq L_{\delta} \cdot \delta(t)+c_{k} \int_{0}^{t}|y(s)-x(s)| d s .
\end{aligned}
$$

From the Gronwall-Bellman inequality, we conclude

$$
\begin{aligned}
|y(t)-x(t)| & \leq L_{\delta} \times \delta(t)+\int_{0}^{t}\left[L_{\delta} \times \delta(s) \times c_{k} \exp \left(\int_{s}^{t} c_{k} d t\right)\right] d s \\
& \leq\left[L_{\delta}+L_{\delta}^{2} c_{k} \exp \left(c_{k}\right)\right] \times \delta(t) .
\end{aligned}
$$

From Definition 4, Equation (4) has the Hyers-UlamRassias stability on $[0,1]$.

\section{Example}

In this section, we give two examples to illustrate our main results.

Example 1. Consider the following problem of the CaputoFabrizio fractional differential equation of form

$$
\left\{\begin{array}{l}
x^{\prime}(t)+{ }^{C F} D^{\frac{1}{3}} x(t)=\frac{e^{-t}}{|x|+8}, t \in[0,1], \\
x(1)=I_{0^{+}}^{\frac{1}{2}} x\left(\frac{1}{4}\right),
\end{array}\right.
$$

and the following inequality

$$
\left|y^{\prime}(t)+{ }^{C F} D^{\frac{1}{3}} y(t)-\frac{e^{-t}}{|y|+8}\right| \leq \delta(t), t \in[0,1] .
$$

Let

$$
\beta=\frac{1}{3}, \gamma=\frac{1}{2}, \xi=\frac{1}{4}
$$

Then

$$
M\left(\frac{1}{3}\right)=\frac{6}{5}, a_{\frac{1}{3}}=2, b_{\frac{1}{3}}=\frac{1}{2},
$$

since

$$
k(t, x)=\frac{e^{-t}}{|x|+8},(t, x) \in[0,1] \times \mathbb{R}
$$

Then, it follows that

$$
\begin{aligned}
& \left|k\left(t, x_{1}\right)-k\left(t, x_{2}\right)\right|=e^{-t}\left|\frac{1}{\left|x_{1}\right|+8}-\frac{1}{\left|x_{2}\right|+8}\right| \\
& \leq e^{-t}\left|\frac{1}{\left(\left|x_{1}\right|+8\right)\left(\left|x_{2}\right|+8\right)}\right|\left|x_{1}-x_{2}\right| \\
& \leq \frac{e^{-t}\left|x_{1}-x_{2}\right|}{64} \leq \frac{1}{64}\left|x_{1}-x_{2}\right| \text {. }
\end{aligned}
$$

Hence, $c_{k}=1 / 64$.

Therefore, $\left(S_{1}\right)$ and $\left(S_{2}\right)$ are satisfied, $\xi^{\gamma} /(\Gamma(\gamma+1))+$ $E c_{k}=(1 / 4)^{1 / 2} / \Gamma(1 / 2+1)+3 \times 1 / 64<1$. By Theorems 10 and 11, Equation (4) has a unique solution

$$
x(t)=x(0)+\frac{3}{4} \int_{0}^{t} \exp (-2(t-s)) \frac{e^{-s}}{|x|+8} d s+\frac{1}{4} \int_{0}^{t} \frac{e^{-s}}{|x|+8} d s .
$$

Set $\delta(t)=e^{t} \in C([0,1],(0,+\infty)), \quad \int_{0}^{t} \delta(s) d s=\int_{0}^{t} e^{s} d s=e^{t}$ $-1 \leq e^{t}$; we conclude $L_{\delta}=1>0$. 
Because $y(t)$ satisfies the following inequality:

$$
\left|y^{\prime}(t)+{ }^{C F} D^{\frac{1}{3}} y(t)-\frac{e^{-t}}{|y|+8}\right| \leq \delta(t), t \in[0,1]
$$

it follows that

$$
\begin{gathered}
\mid y(t)-x(0)-\left(1-\frac{b_{1 / 3}}{a_{1 / 3}}\right) \int_{0}^{t} \exp \left(-a_{\frac{1}{3}}(t-s)\right) k(s, y(s)) d s \\
-\frac{b_{1 / 3}}{a_{1 / 3}} \int_{0}^{t} k(s, y(s)) d s \mid \leq e^{t} .
\end{gathered}
$$

Because $\left(S_{1}\right),\left(S_{2}\right)$, and $\left(S_{3}\right)$ are satisfied, by Theorem 13 , it follows that

$$
|y(t)-x(t)| \leq\left[L_{\delta}+L_{\delta}^{2} c_{k} \exp \left(c_{k}\right)\right] \cdot e^{t} \leq\left(1+\frac{1}{64} e^{\frac{1}{64}}\right) \cdot e^{t} .
$$

Consequently, the equation has the Hyers-Ulam-Rassias stability.

Example 2. Consider the following problem of the CaputoFabrizio fractional differential equation of form

$$
\left\{\begin{array}{l}
x^{\prime}(t)+{ }^{C F} D^{\frac{1}{2}} x(t)=\frac{t}{|x|+8}, t \in[0,1], \\
x(1)=I_{0^{+}}^{\frac{1}{3}} x\left(\frac{1}{2}\right),
\end{array}\right.
$$

and the following inequality

$$
\left|y^{\prime}(t)+{ }^{C F} D^{\frac{1}{2}} y(t)-\frac{t}{|y|+8}\right| \leq \varepsilon, t \in[0,1] .
$$

Let

$$
\beta=\frac{1}{2}, \gamma=\frac{1}{3}, \xi=\frac{1}{2}
$$

Then

$$
M\left(\frac{1}{2}\right)=\frac{4}{3}, a_{\frac{1}{2}}=3, b_{\frac{1}{2}}=1
$$

since

$$
k(t, x)=\frac{t}{|x|+8},(t, x) \in[0,1] \times \mathbb{R}
$$

Then, it follows that

$$
\begin{aligned}
\left|k\left(t, x_{1}\right)-k\left(t, x_{2}\right)\right| & =t\left|\frac{1}{\left|x_{1}\right|+8}-\frac{1}{\left|x_{2}\right|+8}\right| \\
& \leq t\left|\frac{1}{\left(\left|x_{1}\right|+8\right)\left(\left|x_{2}\right|+8\right)}\right|\left|x_{1}-x_{2}\right| \\
& \leq \frac{t\left|x_{1}-x_{2}\right|}{64} \leq \frac{1}{64}\left|x_{1}-x_{2}\right| .
\end{aligned}
$$

Hence, $c_{k}=1 / 64$.

Therefore, $\left(S_{1}\right)$ and $\left(S_{2}\right)$ are satisfied, $\xi^{\gamma} /(\Gamma(\gamma+1))+$ $E c_{k}=(1 / 2)^{1 / 3} / \Gamma(1 / 3+1)+3 \times 1 / 64<1$. By Theorems 10 and 11, Equation (4) has a unique solution

$$
x(t)=x(0)+\frac{2}{3} \int_{0}^{t} \exp (-3(t-s)) \frac{s}{|x|+8} d s+\frac{1}{3} \int_{0}^{t} \frac{s}{|x|+8} d s
$$

Set $y(t)=e^{t} \in C([0,1],(0,+\infty))$, and fix $\varepsilon=9 / 32$; it follows that

$$
\left|y^{\prime}(t)+{ }^{C F} D^{\frac{1}{2}} y(t)-\frac{t}{|y|+8}\right| \leq \frac{9}{32}=\varepsilon, t \in[0,1]
$$

Because $\left(S_{1}\right)$ and $\left(S_{2}\right)$ are satisfied, by Theorem 12, we conclude

$$
|y(t)-x(t)| \leq \exp \left(\frac{1}{64}\right) \cdot \frac{9}{32}=\exp \left(\frac{1}{64}\right) \cdot \varepsilon
$$

Consequently, the equation has the Hyers-Ulam stability.

\section{Conclusions}

In this article, we established the Ulam stability of the Caputo-Fabrizio fractional differential equation with an integral boundary condition by the Laplace transform method. Krasnoselskii's fixed point theorem and Banach fixed point theorem are employed to prove the existence and uniqueness results of the solution for the CaputoFabrizio fractional differential equation. Besides, we constructed a solution for the equation via new Green's function $G(t, s)$. The Ulam stability of the Caputo-Fabrizio fractional differential equation is used to study unusual irregularities and nonlinearities in wave dynamics and liquids motions. Because the Ulam stability is widely used, we will study the Ulam stability of the $\mathrm{ABC}$ fractional differential equation in the future study.

\section{Data Availability}

No data were used to support this study. 


\section{Conflicts of Interest}

The author declares that she has no competing interests.

\section{Authors' Contributions}

The author read and approved the final manuscript.

\section{References}

[1] S. M. Ulam, A collection of the mathematical problems, Interscience, New York, 1960.

[2] D. H. Hyers, "On the stability of the linear functional equation," Proceedings of the National Academy of Sciences of the United States of America, vol. 27, no. 4, pp. 222-224, 1941.

[3] T. M. Rassias, "On the stability of the linear mapping in Banach spaces," Proceedings of the American Mathematical Society, vol. 72, no. 2, pp. 297-300, 1978.

[4] M. Obloza, "Hyers stability of the linear differential equation," Rocznik Naukowo-Dydaktyczny. Prace Matematyczne, vol. 13, pp. 259-270, 1993.

[5] T. Cemil and B. Emel, "Hyers-Ulam-Rassias stability for a first order functional differential equation," Journal of Mathematical and Fundamental Sciences, vol. 47, no. 2, pp. 143-153, 2015.

[6] J. Wang, M. Feckan, and Y. Zhou, "Ulam's type stability of impulsive ordinary differential equations," Journal of Mathematical Analysis and Applications, vol. 395, no. 1, pp. 258264, 2012.

[7] D. Otrocol and V. Ilea, "Ulam stability for a delay differential equation," Central European Journal of Mathematics, vol. 11, no. 7, pp. 1296-1303, 2013.

[8] J. Huang and Y. Li, "Hyers-Ulam stability of delay differential equations of first order," Mathematische Nachrichten, vol. 289, no. 1, pp. 60-66, 2016.

[9] A. Zada, S. Faisal, and Y. Li, "Hyers-Ulam-Rassias stability of non-linear delay differential equations," Journal of Nonlinear Sciences and Applications, vol. 10, no. 2, pp. 504-510, 2017.

[10] S. Wang and F. Meng, "Ulam stability of n-th order delay integro-differential equations," Mathematics, vol. 9, no. 23, pp. 3029-3045, 2021.

[11] J. A. Sabatier, O. P. Agrawal, and J. T. Machado, Advances in fractional calculus: theoretical developments and applications in physics and engineering, Springer, Dordrecht, Netherlands, 2007.

[12] S. D. Eidelman and A. N. Kochubei, "Cauchy problem for fractional diffusion equations," Journal of Differential Equations, vol. 199, no. 2, pp. 211-255, 2004.

[13] A. Kilbas, H. Srivastava, and J. Trujillo, Theory and applications of fractional differential equations, Elsevier Science B.V, Amsterdam, 2006.

[14] A. A. Kilbas and J. J. Trujillo, "Differential equations of fractional order: methods results and problem-i, applicable analysis," An International Journal, vol. 78, pp. 153-192, 2001.

[15] I. Podlubny, Fractional differential equations, Academic Press, San Diego, 1999.

[16] B. Ahmad and S. K. Ntouyas, "A fully hadamard type integral boundary value problem of a coupled system of fractional differential equations," Fractional Calculus and Applied Analysis, vol. 17, no. 2, pp. 348-360, 2014.
[17] B. Ahmad and S. K. Ntouyas, "Existence results for a coupled system of Caputo type sequential fractional differential equations with nonlocal integral boundary conditions," Applied Mathematics and Computation, vol. 266, pp. 615-622, 2015.

[18] J. Henderson, R. Luca, and A. Tudorache, "On a system of fractional differential equations with coupled integral boundary conditions," Fractional Calculus and Applied Analysis, vol. 18, no. 2, pp. 361-386, 2015.

[19] S. Abbas, M. Benchohra, J. Lagreg, A. Alsaedi, and Y. Zhou, "Existence and ulam stability for fractional differential equations of Hilfer-Hadamard type," Advances in Difference Equations, vol. 2017, no. 1, 2017.

[20] J. Wang, L. Lv, and Y. Zhou, "Ulam stability and data dependence for fractional differential equations with caputo derivative," Electronic Journal of Qualitative Theory of Differential Equations, vol. 63, no. 63, pp. 1-10, 2011.

[21] Q. Dai, R. Gao, Z. Li, and C. Wang, "Stability of Ulam-Hyers and Ulam-Hyers-Rassias for a class of fractional differential equations," Advances in Difference Equations, vol. 2020, no. $1,2020$.

[22] K. Liu, M. Fečkan, D. O’Regan, and J. R. Wang, “Hyers-Ulam stability and existence of solutions for differential equations with Caputo-Fabrizio fractional derivative," Mathematics, vol. 7, no. 4, pp. 333-346, 2019.

[23] D. Goufo and E. Franc, "Application of the Caputo-Fabrizio fractional derivative without singular kernel to Korteweg-de Vries-Burgers equation*," Mathematical Modelling and Analysis, vol. 21, no. 2, pp. 188-198, 2016.

[24] J. Losada and J. Nieto, "Properties of a new fractional derivative without singular kernel," Progress in Fractional Differentiation and Applications, vol. 1, pp. 87-92, 2015.

[25] K. S. Miller and B. Ross, An introduction to the fractional calculus and fractional differential equations, Wiley-Interscience, New York, 1993.

[26] J. L. Schiff, The Laplace transform: theory and applications, Springer, New York, 1999.

[27] M. Caputo and M. Fabrizio, "A new definition of fractional derivative without singular kernel," Progress in Fractional Differentiation and Applications, vol. 1, pp. 73-85, 2015. 